\title{
A Systematic Review of Online Sex Addiction and Clinical Treatments Using CONSORT Evaluation
}

\author{
Manpreet K. Dhuffar ${ }^{1}$ - Mark D. Griffiths ${ }^{1}$
}

Published online: 15 April 2015

(C) Springer International Publishing AG 2015

\begin{abstract}
Researchers have suggested that the advances of the Internet over the past two decades have gradually eliminated traditional offline methods of obtaining sexual material. Additionally, research on cybersex and/or online sex addictions has increased alongside the development of online technology. The present study extended the findings from Griffiths' (2012) systematic empirical review of online sex addiction by additionally investigating empirical studies that implemented and/or documented clinical treatments for online sex addiction in adults. A total of nine studies were identified and then each underwent a CONSORT evaluation. The main findings of the present review provide some evidence to suggest that some treatments (both psychological and/or pharmacological) provide positive outcomes among those experiencing difficulties with online sex addiction. Similar to Griffiths' original review, this study recommends that further research is warranted to establish the efficacy of empirically driven treatments for online sex addiction.
\end{abstract}

Keywords Internet sex addiction · Cybersex · Cybersex addiction $\cdot$ Internet-enabled sexual addiction $\cdot$ Behavioral addiction $\cdot$ Online $\cdot$ Technology $\cdot$ Online sex

This article is part of the Topical Collection on Technology and Addiction

Manpreet K. Dhuffar

manpreet_dhuffar@hotmail.co.uk

1 Psychology Division, Nottingham Trent University, Burton Street, Nottingham NG1 4BU, UK

\section{Introduction}

The Internet can be understood as a medium that provides greater access to a plethora of affordable sexual material (e.g., pornography) and the exchange of sexual content (e.g., enhancement of sexual arousal through cybersex) in an anonymous setting $[1,2]$. Recent research has referred to sex addiction as a toxic trilogy, namely 'The 3C's,' (chronicity, content, and culture; [3]). In the last decade, cybersex addiction has commonly been referred to as the "crack cocaine" of sex addiction [4-6]. Because of the almost instant gratification, Internet pornography and/or cybersex provide another outlet to engage in sexually addictive behavior that has the potential of rapid escalation $[2,5]$.

Additionally, there are a variety of positive impacts that Internet usage has had on the experiences of individual sexuality (globally), especially women [7] and youth [8], as well as exceptionally marginalized populations, such as gay [9], lesbian [10], bisexual [11], transgendered individuals [12], and the disabled [13]. The major benefit for these groups includes the fact that the Internet provides a "safe" space for sexual exploration as being online poses less physical and social danger than offline activities $[14,15]$. However, such technological developments have given rise to problematic Internetenabled sexual behavior and has led to a minority of individual's interpersonal relationships, work productivity, and academic success being negatively affected $[16 \bullet \cdot]$. Although prevalence rates of online sex addiction (OSA) remain unclear among the general population, pornography viewing online is presumed to be the most widespread form of cybersex-at least for males $[17,18 \bullet, 19]$.

\section{Defining Online Sex Addiction}

Definitions of OSA (and its derivatives) have remained inconsistent, in part because there is no universal agreement on 
either offline sex addiction or Internet addiction itself. Before examining the empirical literature concerning Internet sex addiction, it is imperative to draw upon the theoretical framework associated with it $[20 \bullet \cdot$. Based on the current framework, Griffiths $[20 \bullet$ ] noted that "Internet sex addiction may not merely be a sub-form of Internet addiction, but also a subform of sex addiction" (p. 112). Such a formulation potentially presents difficulties in conceptualization, definition, and diagnosis.

Over the last 30 years, the ongoing debates about sexual addiction and/or hypersexual disorder have led much disagreement among scholars and clinicians. The latest (fifth) edition of The Diagnostic and Statistical Manual of Mental Disorders (DSM-5; American Psychiatric Association [21]) re-classified Gambling Disorder from a disorder of impulsive control to a behavioral addiction. The only other potentially addictive disorder that was considered for inclusion in the DSM-5 was Internet Gaming Disorder [22-24] and was included in Section III ("Emerging Measures and Models"). These inclusions suggest there is no theoretical reason why sex addiction could not appear in future editions of the DSM. However, while problems related to Internet-related sexuality is of growing concern, the APA concluded that there was insufficient evidence supporting the inclusion of generalized Internet addiction or other sub-types of Internet addiction (such as cybersex addiction and social networking addiction) in the latest edition of the DSM.

In order to define OSA, it is firstly essential to define sex addiction and/or hypersexual disorder. Previous symptomatic and diagnostic definitions have adopted the language of $D i$ agnostic and Statistical Manual of Mental Disorders-IV-TR (DSM-IV-TR) and replaced the word "substance" with the words "sexual behavior." Carnes [25] argued that an ongoing pattern of uncontrolled sexual behavior warranted the term sexual addiction because it comprised "a pathological relationship with a mood altering substance" (p. 4.). More recently, "hypersexual disorder" (HD) has been conceptualized as primarily a non-paraphilic sexual desire disorder with an impulsivity component [26]. HD was considered as a potential disorder within the DSM-5 (in which cybersex as a specifier was also included). However, the proposal to include it was subsequently rejected by APA [21]. While the present authors acknowledge that OSA can potentially be explained from a number of viewpoints, we are also mindful that HD is a recent phenomenon. For the purpose of this review, the present authors concur with the view that the idiosyncrasies of sex addiction (both online and offline) remain the same, regardless of what it is called [27, 28].

\section{Empirical Research into Online Sex Addiction}

In relation to sex online, Griffiths [20••] carried out a comprehensive review of empirical research investigating Internet sex addiction among the adult populations. In that review, only 14 empirical studies of Internet sex addiction in adults were identified that met all of the inclusion criteria (nine quantitative studies [29-37] and five qualitative studies [28-41]).

Most notably, findings obtained from four quantitative studies based on the data collected by Cooper et al. [30-33] were considered fundamental as they provide an empirical overview of problematic OSA. The four published studies by Cooper and colleagues all came from the same participants, and data were collected via the American $M S N B C$ website. Each of the studies examined slightly different areas of OSA. The first study [30] investigated the characteristics and patterns of online sexual behaviors in a sample of 9265 adults (mean age 34 years) through self-report measures. The criteria assessed for cybersex compulsion included the following: time spent online for sexual pursuits, tolerance, harm, denial, unsuccessful abstinence efforts, continuation in spite of negative consequences, interference with life, obsession, and compulsion. Based on self-report scores, participants were categorized as non-sexually compulsive (NSC; $n=7728$ ), moderately sexually compulsive (MSC; $n=1007$ ), sexually compulsive (SC; $n=424)$, and cybersexually compulsive (CSC; $n=96$ ).

The first three groups spent an average of $5 \mathrm{~h}$ a week on the Internet for sexual purposes compared to the cybersex compulsive group that spent $20 \mathrm{~h}$ weekly. With respect to usage patterns, the CSC group had a preference for chat rooms (70 \% females, $43 \%$ males) and the web (36\% males, $10 \%$ females) compared to other Internet applications. A key finding was that the CSC group was significantly more likely to include females, bisexuals and homosexuals, singles daters, and students. Furthermore, $24 \%$ of the CSC group experienced total life interference, and $9 \%$ reported that their behaviors impacted all areas of their lives, namely personal, occupational, social, and recreational areas. Finally, the study reported that higher sexual sensation seeking (i.e., the tendency to take part in new or dangerous sexual activities) differentiated the CSC and the SC from the other two groups [30]. This verifies the implicit assumption that CSCs and SCs are more likely to explore their sexualities relative to the other groups.

The following two studies $[31,32]$ included the same sample of 7037 adults, $84 \%$ of which were male. The aims of Cooper et al.'s [31] study were to assess online sexual problems (OSPs) and to identify their predictors. Those with OSPs ( $6 \%$ of the total sample) were compared to those with no online sexual problems (NOSP). An OSPs was defined as "the use of computerized content (text, sounds, or images obtained from computer software or the Internet or any combination of these) for sexual stimulation and gratification that seems to cause the user difficulty and be perceived as beyond his or her control" (p. 269). The OSP group was found to differ significantly from the NOSPs in that they spent more time online and used the Internet to cope with their offline problems and to explore sexual fantasies. Moreover, the OSPs 
group masturbated online significantly more frequently than the NOSPs group. The NOSPs group used the Internet significantly more often for buying sex materials and for educational purposes.

Finally, the OSPs group scored significantly more in viewing the Internet as problematic, feeling that it was out of control, being addicted to the Internet (a group total of $13.3 \%$ ), to sex $(21.1 \%)$, or both Internet and sex (33.3\%; [33]). Compared to the first study, this study used no assessment of sexual compulsivity. Instead, the authors included an OSA questionnaire [42] that was used in the other previous study. Additionally, the authors themselves highlighted that there was no selfreport to specifically measure Internet sexual compulsivity [42]. Developing such an instrument appears to be much needed for any future research in the field.

The third study examined potentially problematic OSA. The problems of the previous study with regard to the measurements utilized [32] were addressed as authors included an assessment of sexual compulsivity [43]. In terms of user characteristics, the results indicated that six times more males engaged in OSA compared to females and they preferred different Internet applications for sex: $68 \%$ of men used websites compared to $50 \%$ of females, whereas females used chat rooms preferably relative to males (26\% compared to $13 \%)$. Finally, $10 \%$ of men engaged in newsgroups compared to only $4 \%$ of females. A notable finding that came out of this study was the formation of the distinction between SC and at-risk users [31].

The final study conducted by Cooper et al. [33] investigated the reasons for OSA and preferred Internet applications in 384 men who had OSP. Reported motivations for OSA included distraction ( $81 \%$ ), coping (57\%), pursuing sexual activities that would not be done offline (43\%), for educational purposes $(25 \%)$, for socialization (16\%), meeting offline sex partners (12\%), meeting offline dates (9\%), getting support for sexual concerns $(8 \%)$, and buying sexual materials (6\%). The preferred application was the Web that was used for browsing sexual content. Furthermore, the study found two dimensions of problem OSA that can be distinguished in terms of the purpose of the activity: the improvement and the substitution of embodied sex. Each of these may be related to the pathological expression of Internet sex [32]. The findings of this particular study add to the insights from the other three studies conducted [30-32] in that they particularly target men with online sexuality problems, who appear to be an atrisk population for developing sexual compulsivity [32].

Overall, the 14 studies reviewed by Griffiths [20••] highlighted the essential characteristic that differentiated people who engage in cybersex in a healthy and complementary way to their offline sexuality was not excessive use per se, but the presence of negative consequences. From a diagnostic point of view, the excessive engagement in sex may be viewed as a genuine psychopathological disorder once it causes significant impairment in a person's life (e.g., professional, social, romantic, and leisure life [44]). Additionally, if cybersex users experience clinically significant distress or impairment due to their engagements in sexual behaviors on the Internet, it may be indicative of cybersex addiction. This has significant implications for the management of the disorder because addressing the consequences that result from cybersex addiction in treatment (such as cognitive -behavioral therapy $[\mathrm{CBT}]$ ) may prove beneficial in alleviating the symptoms associated with it $[20 \bullet \cdot$. Therefore, the purpose of the current paper is to systematically review and expand upon Griffiths' original review and examine studies that document clinical treatment interventions for OSA in adults.

The present review therefore focuses on the treatment of problematic online sex as this aspect was not examined in Griffiths' previous systematic review. Additionally, the present paper aims to appraise the quality of included studies using the Consolidating Standards of Reporting Trials (CONSORT) criteria that are recognized as a 'gold standard' for assessing and the reporting quality of clinical trials [45]. The CONSORT criteria are not limited to randomized control trials (RCTs) and have been used previously in behavioral addiction research to evaluate the efficacy of outcome studies $[45,46]$. To assess the quality of each individual study, the CONSORT checklist was used as a template to measure overall compliance. As highlighted by King et al. [45], "it should also be noted that the review does not differentiate between the most efficacious treatment available" (p. 1111) for cybersex addiction. In line with King et al. [45], the aim was to evaluate the studies in terms of its adherence to the relevant CONSORT criteria of reporting quality (i.e., how well the treatment approach adopted was described, how well it was administered, and by whom). However, identification of higher quality treatment studies can potentially be of some benefit to practitioners and for providing a base on which future studies can be developed [45].

\section{Method}

\section{Framework for Data Synthesis}

A narrative synthesis approach was employed due to the diversity of methodology in each study. Such an approach provides synthesis of evidence relevant to a wide range of questions including (but not restricted to) effectiveness that relies primarily on a textual approach to summarize and explain - to "tell the story" of - the findings of multiple studies [47].

How a narrative synthesis is conducted varies widely [48]. Traditionally, there has been a lack of consensus as to the basic elements of the approach or the conditions for establishing credibility. A guidance of this method of synthesis was developed by the Economic and Social Research Council (ESRC) 
on the ways in which to conduct a narrative synthesis in systematic reviews [49-51]. This guidance offers both a general framework and specific tools and techniques that aid in increasing transparency of this type of synthesis.

The general framework of Narrative Synthesis comprises four elements: (i) developing a theory of how the intervention works, why, and for whom; (ii) developing a preliminary synthesis of findings of included studies (tabulation, grouping and clusters, transforming data); (iii) exploring relationships within and between studies (qualitative case descriptions, visual representation of relationship between study and characteristics); and (iv) assessing the robustness of the synthesis (reflecting critically, conclusions, and final recommendations).

Although the framework is divided into these four elements, the elements themselves do not have to be undertaken in a strictly sequential manner nor are they totally independent of each other. The reviewers are likely to move iteratively among the activities that make up these four elements.

\section{Inclusion Criteria}

Studies that reported psychological and/or pharmacological treatment interventions for OSA (and its derivatives as outlined below) were considered using the following inclusion criteria: The study (i) reported treatment outcomes for cybersex addiction (including qualitative studies and clinical case studies), (ii) made reference to treatment among adult populations (including young adults), (iii) was published between 1995 and 2014 (since the term OSA did not exist prior to 1995), (iv) did not evidence OSA as a consequence of axis I or axis II disorders (e.g., bipolar disorder), and (v) reported new empirical data.

\section{How Relevant Literature Was Identified}

Cyber activities and related technologies evolve rapidly, thus presenting issues for researchers with regard to use and evaluation of the most current platforms. This further complicates rigorous screening and evaluation. A comprehensive literature search was used to identify relevant studies. Since there is a dearth in studies reporting outcomes of a treatment intervention, literature was identified by searching for publications from January 1995 to September 2014 in the database Web of Science, EBSCOhost-Academic Search Complete: MEDLINE; PsycINFO; PsychARTICLES; Dissertation Abstracts International, searched through ProQuest Dissertation Express; EThOs-British Library; Cochrane Central Register of Controlled Trials; and Campbell Collaboration and through consultations with experts in the field. Previous reviews of the literature $[20 \bullet \bullet, 52,53,54 \bullet \bullet, 55 \bullet]$ were also used to identify additional relevant studies. Only papers reported in English were considered. Most frequent search terms included "cybersex" or "online sex addiction" AND "treatment" OR "pharmacotherapy." Other search terms included "online sex addiction," "cybersex addiction," "internet sex addiction," "internet-enabled sex addiction," "problem cybersex," "problematic online sex," "excessive online sex," "compulsive cybersex," "out-of-control online sex," "compulsive online sex," "obsessive cybersex," "treatment," "university students," "college students," "cognitive -behavioral therapy," "self-help," and "counselling."

The database yielded a total of 1997 citations. All citations were screened based on title and abstracts to assess suitability using a data extraction form (designed specifically for rigorous synthesis of each study). The syntheses and selection process was performed by the first author. Studies that met the criteria were read twice to ensure accurate inclusion and topic relevance. Uncertainty was resolved with the second author. After synthesis of full-text articles, nine studies that documented the treatment of OSA were identified (see Fig. 1). Studies presented in the subsequent section are those that were deemed most significant to the field because they extend previous work and/or offer new empirical information on cybersex addiction treatment.

\section{Quality Assessment}

Studies included within the review were evaluated using the 2010 guidelines of the CONSORT statement. The measurement of compliance followed a two-point grading system, similar to the system used by King et al.'s review [46]. Each item was given a maximum score of " 2 ." A score of " 0 " was given if the item was not present at all; a "1" if it was partially present, and a " 2 " if the CONSORT item was presented with adequate justification. To demonstrate the scoring method, the CONSORT item 20 states "Trial limitations, addressing sources of potential bias, imprecision, and, if relevant, multiplicity of analyses." A score of 0 would be given if the trial did not address limitations; a score of " 1 " would be given if the researchers provided details on some limitations but did not report potential bias; and a score of " 2 " would be given if the researchers clearly described the sources of potential bias, imprecision, and multiple analyses (where necessary). Furthermore, in cases where the CONSORT item was not present due to limitations of the study design, a score of "0" on that item was given and a total score for each study was obtained.

In terms of the methodological approach for each study, the CONSORT checklist assessed whether any of the nine included studies reported (i) a structured summary of design, methods, and results; (ii) the eligibility criteria for participants; (iii) concise details of the intervention; and (iv) a justification for the sample size. Although failure to report such details resulted in lower levels of compliance as highlighted in the CONSORT, the studies were not excluded. 
Fig. 1 Flowchart of review process

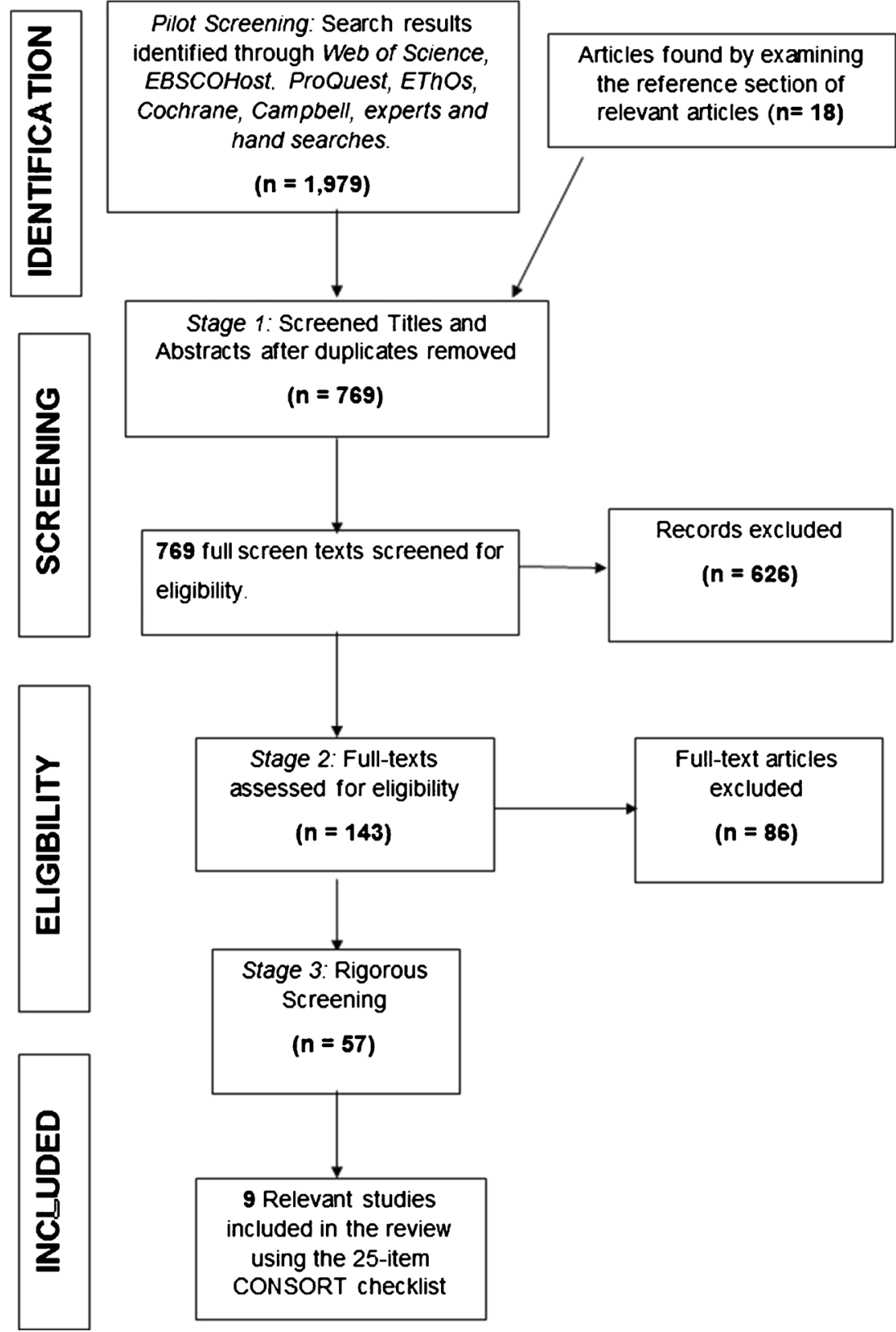

homogeneity between study variables could be examined more closely. This enabled the evaluative process of assessing the differential impacts of treatment for online sex addiction. More specifically, the subgroup analyses were undertaken so that interactions between treatment outcomes could be better explored and thus determining the efficacy of the intervention provided.

\section{Heterogeneity Between Studies}

The four main differences between study variables included (i) type of design, (ii) assessment, (iii) definition and diagnosis, and (iv) types of treatment approaches. This was the primary reason for as to why meta-analysis was not performed.
Subgroup analysis involved the drawing up of clusters by identifying groups and relationships between emerging theme similarities. Findings were organized so heterogeneity and 


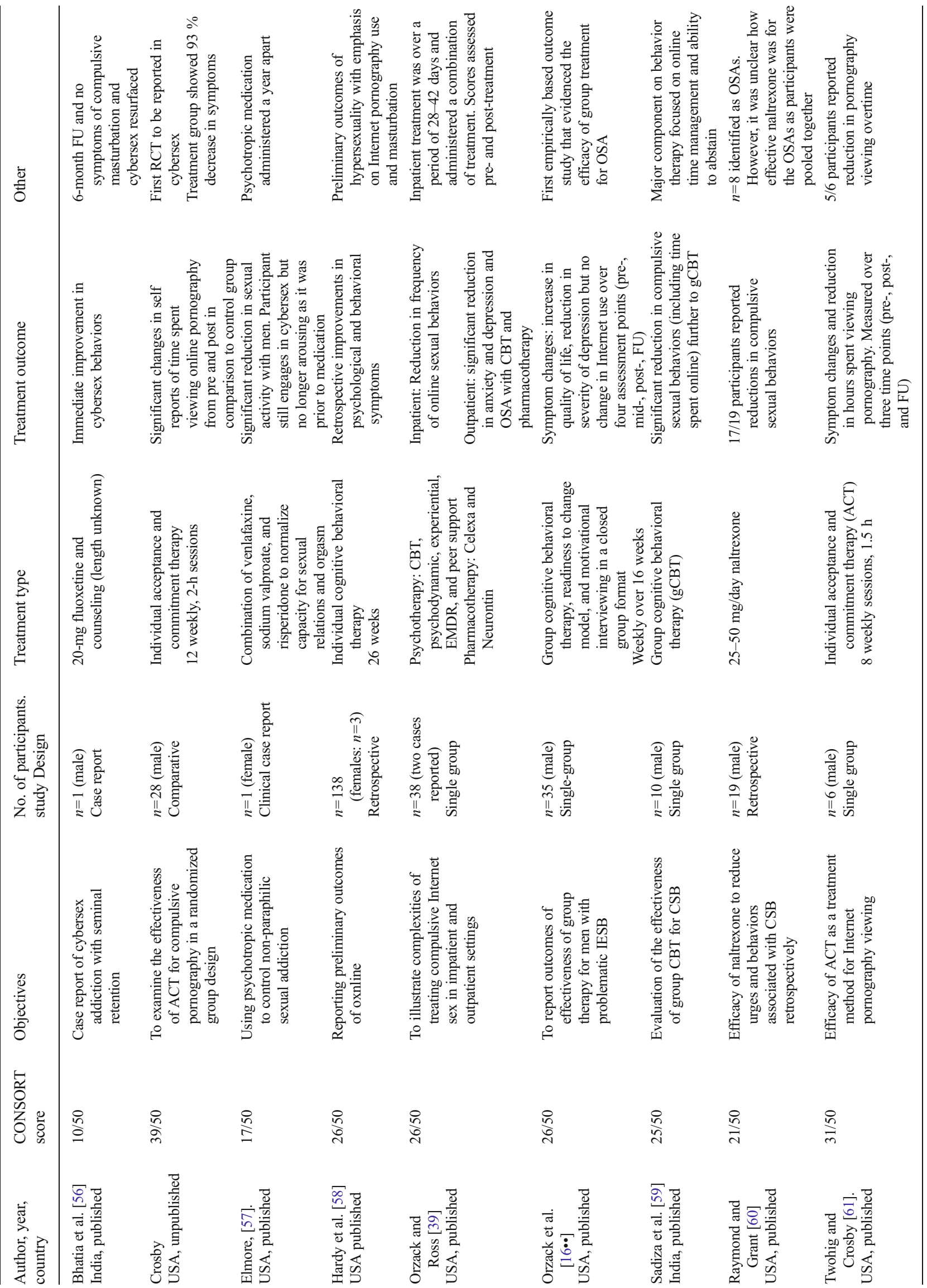


Often, specific inclusion criteria are sought at the initial stages of the review in order to reduce significant differences among studies. Given the limited number of studies implementing interventions for OSA, the approach to the current review was exploratory and was to generate as much evidence as possible prior to the refinement and exclusion process. The main heterogeneous variables are outlined below.

\section{Study Design}

The research designs varied across studies and included retrospective designs $(n=2)$; single case studies $(n=2)$; singlegroup pre-, post-treatment, and follow-up designs $(n=4)$; and comparative designs $(n=1)$. The total number of participants in the nine studies was 276 (four females).

\section{Assessment Tools}

All studies used a range of self-report measures to assess for OSA and associated psychological distress. Assessment tools for OSA included Orzack Internet Addiction Measure [62], Sexual Compulsivity Scale [43], Sexual Symptom Assessment Scale, and Behavioral and Symptom Identification Scale-32 [63]. A self-report measure of consequences associated with compulsive pornography use was used in one of the studies [64].

Measurements of psychological functioning included the following: Self -monitoring, Beck Hopelessness Scale, Beck Depression Inventory, Beck Depression Inventory-II, StateTrait Anxiety Inventory, Perceived extent of recovery (behavioral and psychological), Clinical Global Impression, Obsessive-Compulsive Inventory, and Thought Action Fusion Scale.

\section{Diagnosis and Definition}

All nine included studies referred to the negative consequences associated with OSA use in their introductory sections, but the operational definitions of OSA used to diagnose all varied to some degree. For example, Orzack and Ross [39] adopted Goodman's [65] definition of sexual addiction to explain the sexual components of OSA: a maladaptive pattern of sexual behavior, leading to clinically significant impairment or distress, as manifested by three or more of the following, occurring at any time in the same 12-month period: (1) Tolerance: (a) a need for markedly increased amount of or intensity of the sexual behavior to achieve the desired effect; (b) markedly diminished effective with continued involvement in the sexual behavior at the same level of intensity. (2) Withdrawal: (a) Characteristic psychophysiological withdrawal syndrome of physiologically or psychologically described in the changes upon discontinuation of the behavior; (b) the same or a closely related sexual behavior is engaged in to relieve or avoid withdrawal symptoms. (3) The sexual behavior is often engaged in over a longer period, in a greater quantity, or at a higher level of intensity than was intended. (4) There is a persistent desire or unsuccessful efforts to cut down or control the sexual behavior. (5) A greater deal of time is spent in activities necessary to prepare for the sexual behavior, to engage in the behavior, and to recover from its effects. (6) Important social, occupational, or recreational activities are given up or reduced because of the sexual behavior. (7) The psychological problem that is likely to have been caused or exacerbated by sexual behavior continued despite knowledge of its consequences. Orzack's [62, 66] definition of computer/ Internet addiction was based on the criteria for pathological gambling adapted from the DSM-IV-TR: Evidence of at least five of the following symptoms is indicative of a possible addiction to or dependency on the computer/Internet: (1) Experiencing pleasure, excitement, or relief while on the computer/Internet; (2) spending an ever-increasing amount of time on, and money towards, computer actives with diminished returns; (3) buying the newest and fastest computer hardware; (4) experiencing dysphoric moods while not on the computer/Internet; (5) becoming anxious, angry, or depressed when not on the computer/Internet; (6) feeling a loss of control or being overwhelmed when not on the computer/ Internet; (7) being preoccupied with thoughts about the computer/Internet when not on the computer/Internet; (8) attempting, unsuccessfully, to limit computer/Internet use repeatedly; (9) using the computer/Internet to escape current problems; (10) neglecting daily obligations due to computer/ Internet use; (11) losing significant relationships due to computer/Internet activities; (12) lying about the amount of time spent on the computer/Internet and the content of websites visited; (13) experiencing financial difficulties due to time spent on the computer/Internet; (14) experiencing academic difficulties due to time spent on the computer/Internet; and (15) experiencing physical health problems due to computer/Internet use (p. 349). Raymond and Grant [60] used the term "non-paraphilic compulsive sexual behavior" and characterized it as "recurrent and intense sexually arousing fantasies, sexual urges, and behaviors, which cause individuals distress or impair daily functioning" (p. 57). Crosby used self-selected criteria to define compulsive pornography use that encompassed substance use disorders, impulse control, and obsessive-compulsive spectrum disorder. The variation of definitions adopted in each study could potentially have had an impact in the ways in which OSA was diagnosed.

\section{Types of Interventions}

Studies considered for the final analysis were based on the implementation and administration of a treatment intervention for OSA among the adult population. However, the types of treatment provided varied from study to study. For example, 
Bhatia et al. [56], Elmore [57], and Raymond and Grant [60] administered various types of psychotropic medication to reduce the severity, intensity, and frequency of online sex addiction. For example, in Elmore's study, the female participant was treated with venlafaxine, sodium valproate, risperidone, and paroxetine to normalize her capacity for sexual relations (online and offline) and sexual drive. Whereas Raymond and Grant [60] administered naltrexone $(n=19)$ and Bhatia et al. [56] used a SSRI (fluoxetine) alongside counseling $(n=1)$. The study by Crosby was the only study to report outcomes adopting a RCT administering acceptance and commitment therapy (ACT) as treatment for OSA using a treatment versus control group $(n=28)$. Twohig and Crosby [61] also used $\mathrm{ACT}$ as a treatment method for problematic Internet pornography. Orzack and Ross [39] reported a number of therapeutic modalities (i.e., CBT, psychodynamic, experiential, EMDR, and peer support) to guide residential treatment for OSA. However, outpatient treatment implemented CBT and MET with a combination of psychotropic medication. The most occurring therapeutic treatment administered among the studies was CBT in various forms (e.g., individual CBT [39], online CBT [58], and group CBT [59]). Further details on treatment interventions (i.e., treatment length and outcomes) are summarized in Table 1.

With regard to risk (e.g., physical and emotional harm to self and/or others), only two studies $[60,61]$ provided a review of the limits of confidentiality in their respective groups. Treatment seekers were informed that a report is required in those cases in which clients might be at risk to both self and others (i.e., viewing of images depicting child abuse).

\section{Homogeneity Between Treatment Outcomes}

A number of trends were identified within recurring variables during the analysis process between the included studies. Grouping analyses allowed for identification of the most common and recurring variables across all nine studies. These were then grouped based on their similarities. Recurring outcome variables were categorized into psychological and behavioral outcomes to identify the studies based on whether they measured psychological factors, behavioral factors, or both, relating to the efficacy of treatment for OSA.

\section{Psychological Outcomes}

Significant improvements in overall psychological function following the implementation of a treatment intervention were reported in all studies. Orzack and Ross [39] and Sadiza et al. [59] reported a reduction in symptoms of psychological distress (e.g., anxiety and depression). Orzack et al. [16••] reported significant reductions in symptoms of depression alone, and Bhatia et al. [56] reported reductions in levels of anxiety among the male participant post-treatment and during follow- up. Similarly, Crosby reported improvements in overall psyche and spirituality.

Two studies $[58,61]$ also reported some reduction in obsessive thoughts alongside psychological distress associated with OSA.

\section{Behavioral Outcomes}

Eight studies reported significant reductions and preoccupations (e.g., $[58,61])$ with sexual stimuli, sexual acting out, masturbation (e.g., [57, 59, 61, 66]), and hours spent viewing of online pornography (e.g., [56, 58]). Additionally, Elmore [57] reported there was greater risk reduction (i.e., Internet surfing to meet random men). Orzack et al. [16••] reported an overall improvement in psychological functioning; however, there was no change in Internet use over five assessment points. Finally, Crosby, Orzack et al. [16••], and Twohig and Crosby [61] reported improvements in quality of life.

\section{Direct Impact of Treatment on Cybersexual Behaviors}

All studies (bar two $[16 \bullet \bullet, 57])$ reported positive impacts of treatment (i.e., an overall reduction in cybersexual behaviors) following the implementation of an intervention, irrespective of whether it was individual therapy, group therapy, pharmacotherapy, or peer support. Although the two studies did not assist in the reduction of online sexual behaviors, they did report symptom changes that accompanied the behaviors (e.g., improvement in mood and overall risk). Nevertheless, a majority of the studies reported significant symptom changes reported at post-treatment and/or follow-up stages showed change in the desired direction. While all studies outlined impacts of treatment, respectively, none of them identified specific treatment goals in psychological therapy.

\section{Quality Appraisal}

Using CONSORT, two of the included studies (i.e., [56, 57]) were rated as being of poor quality (10/50 and 17/50). However, it must be noted that both were case studies and assessing them with the CONSORT may have not been appropriate. Five studies $[16 \bullet \cdot, 39,58,59,66]$ were of moderate quality (scores ranged 21-26), and two studies (i.e., Crosby; [61] [Score: $31 / 50]$ ) were of higher quality based on the CONSORT. Since there was only one randomized control trial among all the included studies, it was viewed higher in quality (39/50; Crosby) because it followed a stringent procedure and was more methodologically robust. These analyses are summarized in Table 2. 


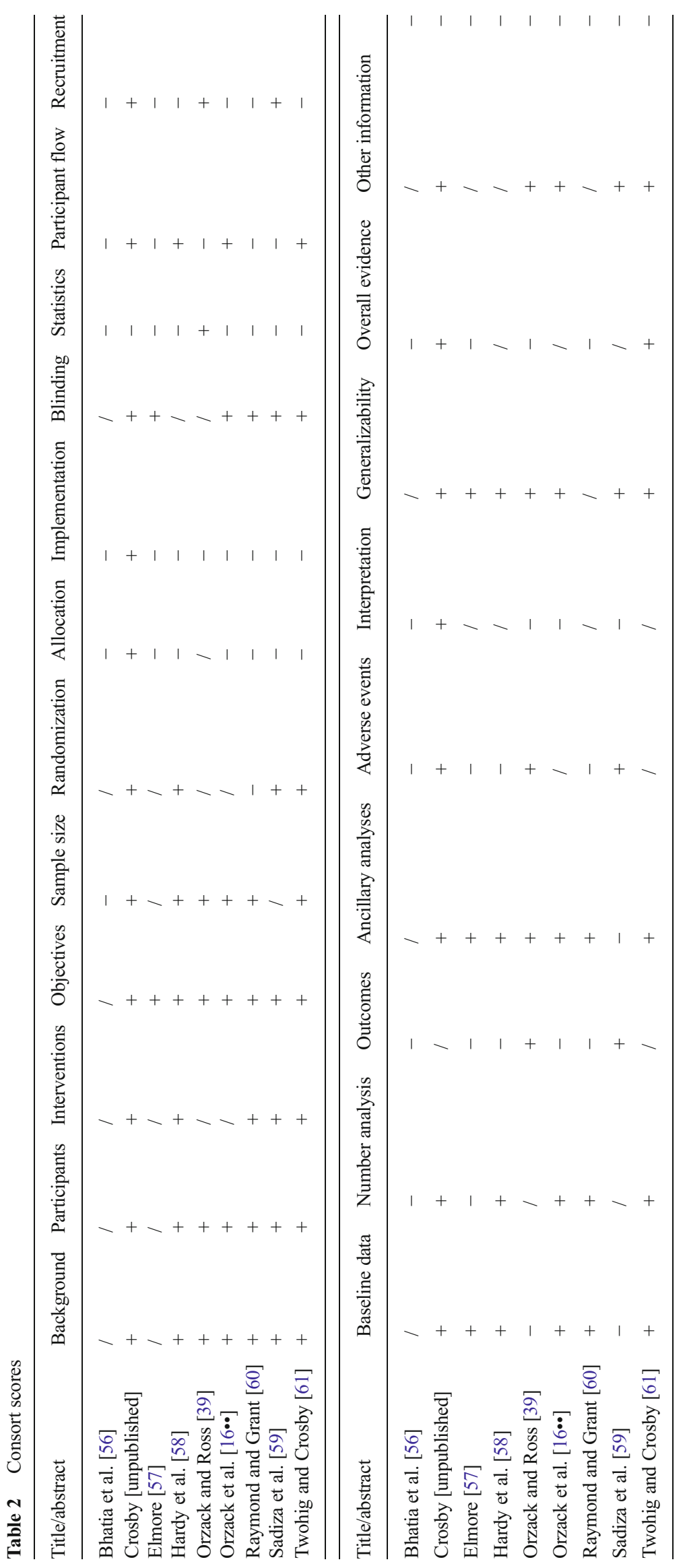




\section{Discussion}

This present paper sets out to systematically explore current treatment interventions available for OSA identified and reported in the psychological literature. Participants across the studies were diagnosed with both online and offline sexual addiction along with other sub-types that included compulsive masturbation and online pornography use. Those with OSA had undergone various treatment regimes in order to reduce symptoms that were associated with their sexually compulsive behaviors. In addition, it is essential to point out that OSA can be considered a genuine problem and this has been evidenced in the studies that implemented a treatment of some kind to those where it has had significant impact upon their lives.

The main findings of this review provided significant support towards the need of the implementation of treatment interventions and empirically supported treatment models for adults that report with hypersexual behaviors online (and the level of risk that is associated with it). Additionally, the present review identified low levels of overall compliance with CONSORT guidelines for reporting clinical trials. Only one of the studies that met the study inclusion criteria included a randomized control design. Most of the studies failed to describe adequate justification for their sample size and did not provide calendar dates of recruitment and intervention.

Interventions for OSA varied considerably across the included studies, with the majority of studies employing both pharmacological interventions and psychological therapy. However, none of the studies identified treatment goals, thus making it unclear as to whether what the overall target of therapeutic success was. Psychotropic and therapeutic duration provided significant variance across each study. The lack of consistency in treating sexual addiction and/or hypersexual disorder and Internet addiction make it difficult to draw any definitive conclusions as to the efficacy of each treatment method. Furthermore, it must be noted that two out of nine studies were based on single cases that make it even more difficult to reach any definitive conclusions regarding efficacy.

Although online sexual habits (as with offline sexual habits) have been described with labels including 'addictive', 'compulsive', and/or 'problematic', it appears that within the nine studies, there was no distinction concerning the content and/or material accessed and viewed. Carnes [67] highlighted that the first point of contact for treatment is typically with a physician. This was evidenced in Bhatia et al.'s [56] case study where the young man initially visited the GP for "seminal retention" issues before actually disclosing he was cybersexually addicted.

Treatment studies into OSA also highlighted that CBTbased on a very small number of studies - appears to be the most effective form of treatment for problematic habitual sex (individually and in a group format) in helping build awareness and aiding in reduction of online sexual behaviors. As highlighted by Weiss [68], a behavioral addiction warrants a behavioral treatment. However, the two studies that employed ACT as a treatment method also highlighted that ACT can be as effective and future studies could feasibly compare both treatment methods with a control group to generate more evidence for OSA. While there was one RCT study that examined ACT, it appears that although CBT is the recommended treatment by many proponents in the field, a RCT to measure its efficacy with a control group is yet to be conducted.

Despite the lack of cohesiveness among researchers, all studies sought to explain OSA, and that treatment could be influenced in the way such a disorder is defined and therefore diagnosed. For instance, the current definitions of sexual addiction and/or hypersexual disorder proposed by Carnes [25], Goodman [65], and Kafka [26] all vary to some degree. Given that OSA can arguably be explained from two viewpoints (i.e., as a sub-type of Internet addiction or as a sub-type of sex addiction), treatment models need to encompass both aspects during psycho-educational stages. This is one way in which treatment perhaps could be more strengthened.

Furthermore, as highlighted by Hardy et al. [58], a common outcome of clinical treatment research is that something is better than nothing, but that most effective treatments do not differ in their degrees of efficiency [69]. However, participant perceptions of recovery and retrospective ratings of symptoms do have considerable validity as measures of important aspects of treatment efficacy and recovery [70, 71].

The empirical research presented in the presented review provides a number of avenues for future treatment interventions to be designed in the area of OSA. The first concerns should address gender, although the present review identified only four women that sought treatment for OSA. The previous literature $[28,30]$ has reported that women, more likely than men, engage in such activities.

\section{Conclusions}

Overall, the studies presented in this paper have highlighted that there are key differences among those who experience online sexual activities in a healthy manner than to those for which cybersex is becomes an addiction and (consequently) seek treatment. In line with the clinical inferences made by Griffiths $[20 \bullet$ ], it appears that behavioral treatments (i.e., CBT) have been proven more beneficial in alleviating such symptoms and, to some extent, negative consequences.

Given that diagnostic criteria are yet to be determined, future treatments could focus on developing an etiological model for hypersexual behaviors and/or sex addiction to shed some light on the specific behaviors that are being addressed [55•]. 


\section{Compliance with Ethics Guidelines}

Conflict of Interest Manpreet Dhuffar declares no conflict of interest. Mark Griffiths has received funding for a number of research projects in the area of gambling education for youth, social responsibility in gambling, and gambling treatment from the Responsibility in Gambling Trust, a charitable body who funds its research program based on donations from the gambling industry. The author also undertakes consultancy for various gaming companies in the area of social responsibility in gambling.

Human Rights and Informed Consent This article does not contain any studies with human or animal subjects performed by any of the authors.

\section{References}

Papers of particular interest, published recently, have been highlighted as:

- Of importance

-• Of major importance

1. Cooper A. Sexuality and the Internet: surfing into the new millennium. CyberPsychol Behav. 1998;1:187-93.

2. Griffiths MD. Sex on the Internet: observations and implications for Internet sex addiction. J Sex Res. 2001;38:333-42.

3. Riemersma J, Sytsma M. A new generation of sexual addiction. Sex Addict Compulsivity. 2013;20:306-22.

4. Cooper A. The Internet and sexuality: into the new millennium. J Sex Educ Ther. 1997;22:5-6.

5. Carnes PJ. Out of the shadows: understanding sexual addiction. 9th ed. Center City, MN: Hazelden; 2001.

6. Adams, K. M. (2008) Cybersex: the crack "cocaine" of sex addiction. The National Psychologist, 6. http://nationalpsychologist.com/ 2008/05/cybersex-the-"crack-cocaine"-of-sex-addiction/101003. html. Accessed, 18 Oct 2014.

7. Leiblum SR. Women, sex and the Internet. Sex Relat Ther. 2001;16:389-405

8. Lou CH, Zhao Q, Gao ES, Shah IH. Can the Internet be used effectively to provide sex education to young people in China? $\mathrm{J}$ Adolesc Health. 2006;39:720-8.

9. McKelland MJ. Virtual ethnography using the Internet to study gay culture in Japan. Sexualities. 2002;5:387-406.

10. Correll S. The ethnography of an electronic bar-the lesbian café. J Contemp Ethnogr. 1995;24:270-98.

11. Koch NS, Schockman HE. Democratising Internet access in the lesbian, gay and bisexual communities. In: Ebo B, editor. Cyberghetto or cybertopia? Race, class and gender on the Internet. Westport, CT: Praeger; 1998. p. 171-84.

12. Broad KL. GLB p T? Gender/sexuality movements and transgender collective identity (de)constructions. Int J Sex Gend Stud. 2002;7: 241-64.

13. Kaufman M, Silverberg C, Odette F. The ultimate guide to sex and disability. San Francisco, CA: Cleis; 2007.

14. Turkle S. Life on the screen. New York, NY: Simon \& Schuster; 1995.

15. Weiss R, Schneider J. Untangling the web. Sex, porn, and fantasy obsession in the Internet age. New York, NY: Alyson; 2006.

16.• Orzack MH, Voluse AC, Wolf D, Hennen J. An ongoing study of group treatment for men involved in problematic Internet-enabled sexual behaviour. CyberPsychol Behav. 2006;9:348-60. This study was the first to empirically report outcomes of group therapy among men that experienced problematic sexual behaviors online.

17. Paul B, Shim JW. Gender, sexual affect, and motivations for Internet pornography use. Int J Sex Health. 2008;20:187-99. doi:10. 1080/19317610802240154.

18. Laier C, Brand M. Empirical evidence and theoretical considerations on factors contributing to cybersex addiction from a cognitive-behavioral view. Sex Addict Compulsivity. 2014;21:305-21. This study is significant because it introduces a cognitive-behavioral model of treatment for cybersex addiction that can potentially be altered to report patient outcomes in the therapeutic process.

19. Shaughnessy K, Byers ES, Walsh L. Online sexual activity experience of heterosexual students: gender similarities and differences. Arch Sex Behav. 2011;40:419-27. doi:10.1007/s10508-010-96299.

20.• Griffiths MD. Internet sex addiction: a review of empirical research. Addict Res Theory. 2012;2:111-24. This study was deemed important because it was the first (contemporary) comprehensive review of empirical research investigating Internet sex addiction.

21. American Psychological Association. Diagnostic and statistical manual of mental health disorders. 5th ed. Washington DC: Author; 2013.

22. Griffiths MD, Kuss DL, Demetrovics Z. DSM-5 Internet gaming disorder needs a unified approach to assessment. Neuropsychiatry. 2014;4(1):1-4.

23. Hertlein KM, Cravens JD. Assessment and treatment of Internet sexuality issues. Current Sex Health Reports. 2014;6:56-63.

24. Petry NM, O'Brien CP. Internet gaming disorder and the DSM-5. Addiction. 2013;108:1186-7.

25. Carnes P. Out of the shadows: understanding sexual addiction. Minneapolis, MN: CompCare; 1983.

26. Kafka MP. Hypersexual disorder: a proposed diagnosis for DSM-V. Arch Sex Behav. 2010;39:377-400.

27. Goodman A. Sexual designation and treatment. J Sex Marital Ther. 1992;18(4):303-14.

28. Schneider JP. A qualitative study of cybersex participants. Gender differences, recovery issues, and implications for therapists. Sex Addict Compulsivity. 2000;7:249-78.

29. Boies SC, Cooper A, Osborne CS. Variations in Internet-related problems and psychosocial functioning in online sexual activities: implications for social and sexual development of young adults. CyberPsychol Behav. 2004;7:207-30.

30. Cooper A, Delmonico DL, Burg R. Cybersex users, abusers, and compulsives: new findings and implications. Sex Addict Compulsivity. 2000;6:79-104.

31. Cooper A, Delmonico DL, Griffin-Shelley E, Mathy RM. Online sexual activity: an examination of potentially problematic behaviours. Sex Addict Compulsivity. 2004;11:129-43.

32. Cooper A, Galbreath N, Becker MA. Sex on the Internet: furthering our understanding of men with online sexual problems. Psychol Addict Behav. 2004;18:223-30.

33. Cooper A, Griffin-Shelley E, Delmonico DL, Mathy RM. Online sexual problems: assessment and predictive variables. Sex Addict Compulsivity. 2001;8:267-85.

34. Daneback K, Ross MW, Mansson SA. Characteristics and behaviours of sexual compulsives who use the Internet for sexual purposes. Sex Addict Compulsivity. 2006;13:53-67.

35. Delmonico DL, Miller JA. The Internet sex screening test: a comparison of sexual compulsives versus non-sexual compulsives. Sex Relat Ther. 2003;18:261-76.

36. Schnarrs PW, Rosenberger JG, Satinsky S, Brinegar E, Stowers J, Dodge B, et al. Sexual compulsivity, the Internet, and sexual behaviors among men in a rural area of the United States. Aids Patient Care and STDs. 2010;24:563-9. 
37. Schwartz MF, Southern S. Compulsive cybersex: the new tearoom. Sex Addict Compulsivity. 2000;7:127-44.

38. Grov C, Bamonte A, Fuentes A, Parsons JT, Bimbi DS, Morgenstern J. Exploring the Internet's role in sexual compulsivity and out of control sexual thoughts/behaviour: a qualitative study of gay and bisexual men in New York City. Cult Health Sex. 2008;10: 107-24.

39. Orzack MH, Ross CJ. Should virtual sex be treated like other sex addictions? Sex Addict Compulsivity. 2000;7:113-25.

40. Schneider JP. The impact of compulsive cybersex behaviours to the family. Sex Relat Ther. 2001;18:329-54.

41. Stein DJ, Black DW, Shapira NA, Spitzer RL. Hypersexual disorder and preoccupation with Internet pornography. Am J Psychiatr. 2001;158:1590-4.

42. Cooper A, Scherer C, Boies SC, Gordon B. Sexuality on the Internet: from sexual exploration to pathological expression. Prof Psychol Res Pract. 1999;30:154-64.

43. Kalichman SC, Johnson JR, Adair V, Rompa D, Kelly JA. Sexual sensation seeking - scale development and predicting aids-risk behavior among homosexually active men. J Pers Assess. 1994;62: 385-97.

44. APA (2010). Hypersexual disorder. DSM-5 development. Retrieved from http://www.dsm5.org/ProposedRevisions/Pages/ proposedrevision. aspx? rid $=415$.

45. Altman DG, Schulz KF, Moher D, Egger M, Elbourne D. The revised CONSORT statement for reporting randomized trials: explanation and elaboration. Ann Intern Med. 2001;134:663-94.

46. King DL, Delfabbro PH, Griffiths MD, Gradisar M. Assessing clinical trials of Internet addiction treatment: a systematic review and CONSORT evaluation. Clin Psychol Rev. 2011;31:1110-6.

47. Popay, J., Roberts, H., Snowden, A., Petticrew., ... Duffy, S. (2006). Guidance on the conduct of narrative synthesis in systematic reviews: a product from the ESRC methods programme.

48. Centres for Reviews and Dissemination. Systematic reviews: CRDs guidance for undertaking reviews in healthcare. York: York Publishing Services Ltd.; 2008.

49. Arai L, Britten N, Popay J, Roberts H, Petticrew M, Sowden A. Testing methodological developments in the conduct of narrative synthesis: a demonstration review of research on the implementation of smoke alarm interventions. Evid Policy. 2007;3:361-83.

50. Popay J, Roberts H, Sowden A, Petticrew M, Britten N, Rodgers M. Developing guidance on the conduct of narrative synthesis in systematic reviews. J Epidemiol Community Health. 2005;59(1):A7.

51. Rodgers M, Sowden A, Petticrew M, Arai L, Roberts H, Westwood M. Testing methodological guidance on the conduct of narrative synthesis in systematic reviews: effectiveness of interventions to promote smoke alarm ownership and function. Evaluation. 2009;15:47-71.

52. Mudry TE, Hodgins DC, el-Guebaly N, Wild T, Schopflocher D. Conceptualizing excessive behaviour syndromes: a systematic review. Curr Psychiatr Rev. 2011;7:138-51.

53. Sussman S, Lisha N, Griffiths M. Prevalence of the addictions: a problem of the majority or minority? Eval Health Prof. 2011;34:356.
54.• Hook JN, Reid RC, Penberthy JK, Davis DE, Jennings DJ. Methodological review of treatments for nonparaphilic hypersexual behavior. J Sex Marital Ther. 2014;40:294-308. This study is significant because it is the first systematic review that evaluates treatment studies of hypersexuality.

55. von Franqué F, Klein V, Briken P. Which techniques are used in psychotherapeutic interventions for nonparaphilic hypersexual behavior? Sex Med Rev. 2014. doi:10.1002/smrj.34. Recent review that extends Hook et al.'s (2014) review on treatments for nonparaphilic hypersexual behavior. Significant to the field because it provides clinical implications relevant to therapists and practitioners.

56. Bhatia MS, Jhanjee A, Kumar P. Seminal retention syndrome with cybersex addiction: a case report. J Clin Diagn Res. 2012;6:879-80.

57. Elmore JL. Psychotropic medication control of non-paraphilic sexual addiction in a female. Sex Relat Ther. 2005;20(2):211-3.

58. Hardy SA, Ruchty J, Hull TD, Hyde R. A preliminary study of an online psychoeducational program for hypersexuality. Sex Addict Compulsivity. 2010;17:247-69.

59. Sadiza J, Varma R, Jena SPK, Singh TB. Group cognitive behaviour therapy in the management of compulsive sex behaviour. Int $\mathrm{J}$ Crim Justice. 2011;6:309-25.

60. Raymond NC, Grant JE. Augmentation with naltrexone to treat compulsive sexual behavior: a case series. Ann Clin Psychiatry. 2010;22:56-62.

61. Twohig MP, Crosby JM. Acceptance and commitment therapy as a treatment for problematic Internet pornography viewing. Behav Ther. 2010;41:285-295.3.

62. Orzack, M. H. (1999). How to recognize and treat computer.com addictions. Directions in Clinical and Counseling Psycbology, Lesson 2, 1999, Vol. 9, pp. 13-26. New York: The Hatherleigh Co.

63. Eisen S, Speredelozzi A. McLean BASIS - 32 instruction manual. Belmont, MA: McLean Hospital Corporation; 2000.

64. McBride KR, Reece M, Sanders SA. Predicting negative outcomes of sexuality using the Compulsive Sexual Behavior Inventory. Int J Sex Health. 2007;19:51-62.

65. Goodman A. A sexual addiction: an integrated approach. Madison, CT: International Universities Press; 1998.

66. Orzack MH. Computer addiction: what is it? Psychiatr Times. 1998;8:32-3.

67. Carnes PJ. Sexual addiction and compulsion: recognition, treatment and recovery. CNS Spectrums. 2000;5:63-72.

68. Weiss, R. (2013, November). Advanced clinical work with porn and sex addiction. CPD Workshop, Day 2, London, UK.

69. Orford J. Asking the right questions in the right way: the need for a shift in research on psychological treatments for addiction. Addiction. 2008;6:875-85.

70. Jensen HH, Mortensen EL, Lotz M. The association between retrospective outcome evaluations and pre-post-treatment changes in psychodynamic group-psychotherapy. Scand J Psychol. 2008;49: 339-43.

71. Pratt CC, McGuigan WM, Katzev AR. Measuring program outcomes: using retrospective pretest methodology. Am J Eval. 2000;21:341-9. 